Classification

Physics Abstracts

$73.00-81.10 \mathrm{~F}-72.20$

\title{
Réalisation et caractérisation d'un transistor à effet de champ JFET au GaAs en vue de son intégration avec une photodiode
}

\author{
M. T. Belaroussi, F. Therez et R. Alcubilla (*) \\ Laboratoire d'Automatique et d'Analyse des Systèmes du C.N.R.S. 7, avenue du Colonel Roche, \\ 31077 Toulouse Cedex, France
}

(Reçu le 10 février 1986, révisé le 23 septembre, accepté le 23 septembre 1986)

\begin{abstract}
Résumé. - Un transistor à effet de champ JFET est fabriqué à partir d'un substrat de GaAs à l'aide de l'épitaxie en phase liquide. La structure comprend deux couches épitaxiées, une zone de GaAs de type $\mathrm{n}$ pour le canal et une région $\mathrm{Ga}_{0,6} \mathrm{Al}_{0,4} \mathrm{As}$ de type $\mathrm{p}$ forme la grille. Un des avantages du dispositif est que le processus de fabrication nécessite seulement quelques opérations. La conception et la technologie de fabrication du composant ainsi que les caractéristiques courant-tension sont décrites. Nous avons obtenu des valeurs de la transconductance égales ou supérieures à $12 \mathrm{~mA} / \mathrm{V}$. Le transistor a montré une tension de seuil de $-4,6 \mathrm{~V}$ et une mobilité des électrons dans le canal atteignant $3850 \mathrm{~cm}^{2} / \mathrm{V}$.s. Les résultats obtenus à l'aide du dispositif précédent seront étendus à la fabrication du circuit intégré GaAlAs-GaAs associant une photodiode à un TEC.
\end{abstract}

\begin{abstract}
A field-effect transistor JFET has been fabricated on a GaAs substrate using Liquid Phase Epitaxy. The structure involves two epitaxial layers of $\mathrm{n}-\mathrm{GaAs}$ for the channel and $\mathrm{p}-\mathrm{Ga}_{0.6} \mathrm{Al}_{0.4} \mathrm{As}$ for the gate. One advantage of the device is that the fabrication process requires only few steps. The design of the device, the fabrication technology and the $I(V)$ characteristics are described. A transconductance value over $12 \mathrm{~mA} / \mathrm{V}$ has been achieved. The transistor have shown a threshold voltage of $-4.6 \mathrm{~V}$ and mobility reaching $3850 \mathrm{~cm}^{2} / \mathrm{V}$.s. The results on the previous device will be applied to the fabrication of GaAlAs-GaAs monolithic integration of a photodiode and FET.
\end{abstract}

\section{Introduction}

L'association d'une hétérojonction et d'un circuit amplificateur à effet de champ sur un même substrat GaAs fait l'objet de plusieurs études en vue des réalisations possibles [1] et de leur optimisation [2, 3]. La conception de tels dispositifs présente l'avantage d'intégrer des composants électroniques et des éléments optiques (photodétecteurs) [4]. L'intégration d'un transistor à effet de champ et d'une diode détectrice élimine les connexions sophistiquées et réduit les capacités parasites. Les performances et la fiabilité sont donc accrues par les dispositifs monolithiques.

Notre étude propose la conception d'un amplificateur FET à jonction associé à un élément photodétecteur. La fabrication du circuit ne nécessite que quelques opérations technologiques. Dans ces conditions, la réussite de l'élaboration du composant dépend avant

(*) Monsieur R. Alcubilla est actuellement à ETSET B. c/Jorge Girona Salgado, s/n 08034 Barcelone, Espagne. tout de la croissance des couches épitaxiées et de la mise en œuvre des opérations de photolithographie. Au cours de ce premier travail, nous nous sommes efforcés de fabriquer et de caractériser le préamplificateur JFET. La partie active du circuit est donc un transistor à effet de champ à hétérojonction qui présente une intensité de grille réduite. Ce courant de saturation est caractéristique de la diode $\mathrm{GaAlAs}-\mathrm{GaAs}$. Une première application du dispositif JFET sera l'augmentation de la sensibilité spectrale de la photodiode. Lorsque la diode $\mathrm{P}-\mathrm{N}$ est intégrée à un TEC, la réponse spectrale de celle-ci est multipliée par la transconductance du préamplificateur.

Le récepteur monolithique considéré présente l'avantage d'utiliser des tensions bien inférieures aux potentiels de claquage des photodiodes à avalanche.

Dans la première partie de la communication, nous décrivons le principe de fonctionnement et la conception du dispositif associant un transistor à effet de champ et un détecteur. La partie suivante est consacrée à la technologie de fabrication du composant en 
mettant l'accent sur les opérations d'élaboration les plus décisives. La dernière partie sur l'étude expérimentale, donne les caractéristiques $I_{\mathrm{DS}}\left(V_{\mathrm{DS}}\right)$ et les courbes de transfert $I_{\mathrm{DS}}\left(V_{\mathrm{GS}}\right)$. L'analyse des résultats expérimentaux précise les valeurs de la transconductance des résistances sur les diverses électrodes et la mobilité des électrons dans le canal.

\section{Principe de fonctionnement et conception du disposi- tif}

Le dispositif décrit est donc un transistor à effet de champ, JFET, associé à une hétérojonction $\mathrm{P}-\mathrm{N}$ détectrice. Le circuit électrique est représenté sur la figure 1. Une augmentation de la tension entre grille et source produit une élévation du courant drain dans un rapport égal à la transconductance $g_{\mathrm{m}}$ du TEC. La tension de grille est contrôlée par la résistance $R_{\mathrm{L}}$ du schéma de la figure 1. Dans ces conditions, l'intensité du courant

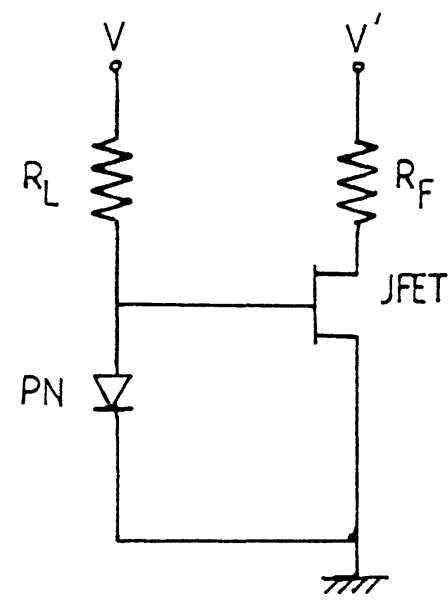

Fig. 1. - Schéma du circuit électrique du dispositif associant un JFET et une photodiode.

[Electric circuit diagram of integrated JFET and photodiode device.]

traversant l'électrode de drain est $g_{\mathrm{m}} \cdot R_{\mathrm{L}}$ fois plus élevée que le courant généré par la photodiode. Il est donc intéressant de réaliser un TEC à transconductance élevée pour un bon fonctionnement du circuit électronique. L'étude de la faisabilité de la partie active du circuit correspond au dessin des gravures des électrodes source, drain et grille de la figure 2 .

Le dessin du contact de source suivant deux régions identiques permet de diviser le composant. Chaque zone présente une longueur égale à $Z / 2=200 \mu \mathrm{m}$. La transconductance $g_{\mathrm{m}}$ est proportionnelle à $Z$. La largeur de la grille est fixée à $200 \mu \mathrm{m}$ pour une moitié du dispositif. La valeur de $Z$ est aussi donnée par le critère de conception qui satisfait à un niveau du courant drain

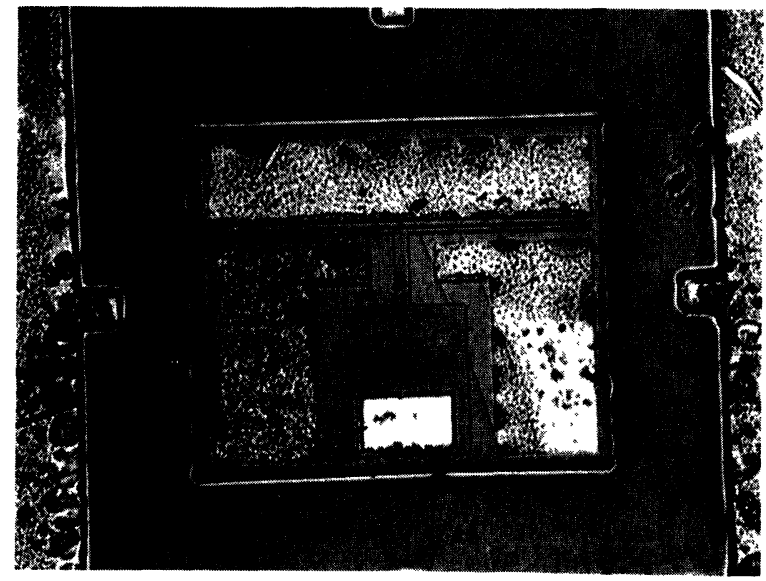

Fig. 2. - Photographie des gravures des métallisations de source de drain et de grille, $L=8 \mu \mathrm{m}, Z=400 \mu \mathrm{m}$; les dimensions du détecteur sont de $160 \mu \mathrm{m}$ par $190 \mu \mathrm{m}$.

[Photograph of source, drain and gate electrodes after selective etching of the metallizations, $L=8 \mu \mathrm{m}, Z=400 \mu \mathrm{m}$; the dimensions of the detector are $160 \mu \mathrm{m}$ and $190 \mu \mathrm{m}$.]

pris égal à environ $30 \mathrm{~mA}$ et une transconductance suffisante.

La longueur de la grille dans le cas de cette première étude est prise égale à $8 \mu \mathrm{m}$. Afin de limiter les valeurs de résistances d'accès de source et de drain, ces deux électrodes sont éloignées de la grille d'une distance de $10 \mu \mathrm{m}$. Les côtés des deux régions de source qui délimitent la connexion entre le TEC et la photodiode sont de $50 \mu \mathrm{m}$. Enfin, les dimensions à l'intérieur de la photodiode (sans le contact métallique) sont de $160 \mu \mathrm{m}$ et $110 \mu \mathrm{m}$ résultant à une surface égale à $1,76 \times 10^{-4} \mathrm{~cm}^{2}$ (les dimensions et la surface extérieures sont respectivement $160 \mu \mathrm{m}$ et $110 \mu \mathrm{m}$ résultant à une surface égale à $1,76 \times 10^{-4} \mathrm{~cm}^{2}$.

Suivant la direction perpendiculaire à la surface, l'épaisseur de la région $\mathrm{n}-\mathrm{GaAs}$ est de l'ordre de $0,6 \mu \mathrm{m}$. Ce dernier paramètre maintient une valeur suffisante de la transconductance ainsi qu'une bonne absorption dans la photoduide. Afin d'assurer un bon compromis entre les paramètres électriques du TEC et la sensibilité spectrale de la photodiode, $N_{\mathrm{D}}$ doit être égal à $2 \times 10^{16} \mathrm{~cm}^{-3}$.

\section{Technologie de fabrication}

Le dispositif associant un transistor à effet de champ et une photodiode est élaboré par la technique d'épitaxie en phase liquide des matériaux GaAlAs. Cette technique est utilisée pour la croissance des couches peu dopées et de bonne qualité obtenue grâce à une concentration de pièges réduite $[5,6]$. Cette méthode de croissance présente en outre une mise en œuvre classique. 
3.1 Conditions D'Élaboration. - Détaillons les particularités du processus technologique pour la croissance de couches uniformes présentant une faible densité de défauts, la structure comprend quatre couches :

- une région tampon formée sur le substrat,

- la couche active du canal n-GaAs,

- l'épitaxie assurant la croissance du p-GaAlAs,

- enfin la couche de contact $\mathrm{p}^{+}-\mathrm{GaAs}$.

Le substrat est du matériau semi-isolant, compensé au chrome et d'orientation $\langle 100\rangle$. La figure 3 montre les vues en coupe du composant après les étapes principales de fabrication.

Les substrats de départ sont nettoyés soigneusement à l'aide de solvants chauffés à $100^{\circ} \mathrm{C}$ et décapés avec la solution $5 \mathrm{H}_{2} \mathrm{SO}_{4}: 1 \mathrm{H}_{2} \mathrm{O}_{2}: 1 \mathrm{H}_{2} \mathrm{O}\left(50^{\circ} \mathrm{C}\right.$, pendant $30 \mathrm{~s})$. qui produit un meilleur état de la surface. Nous avons observé une qualité accrue de nos couches lorsque la croissance est effectuée à partir de bains sursaturés par une réduction de $2{ }^{\circ} \mathrm{C}$ de la température.

Les bains sont saturés à $800^{\circ} \mathrm{C}$. La couche tampon est élaborée lors de la descente en température à partir de celle de décapage égale à $804^{\circ} \mathrm{C}$. Cette croissance se poursuit jusqu'à la sursaturation des bains produite à $798^{\circ} \mathrm{C}$ qui correspond à l'instant où l'échantillon est amené sous le bain contenant de l'étain pour la formation de la couche active n-GaAs. Ce cycle d'épitaxie et le mode opératoire ont été décrits précédemment [7].

Dans ces conditions, les défauts de croissance sont très peu nombreux et confinés à la périphérie de la plaquette. Nous obtenons $N_{\mathrm{D}}=6 \times 10^{16} \mathrm{~cm}^{-3}, N_{\mathrm{A}}=$ $10^{18} \mathrm{~cm}^{-3}$ dans $\mathrm{p}-\mathrm{Ca}_{0,6} \mathrm{Al}_{0,4} \mathrm{As}$ et $5 \times 10^{18} \mathrm{~cm}^{-3}$ atomes de Ge pour le $\mathrm{p}^{+} \mathrm{GaAs}$.

Les épaisseurs des régions $\mathrm{n}-\mathrm{GaAs}$ et $\mathrm{p}-\mathrm{GaAlAs}$ de l'échantillon PI6 sont respectivement égales à $0,4 \mu \mathrm{m}$ et $0,5 \mu \mathrm{m}$.

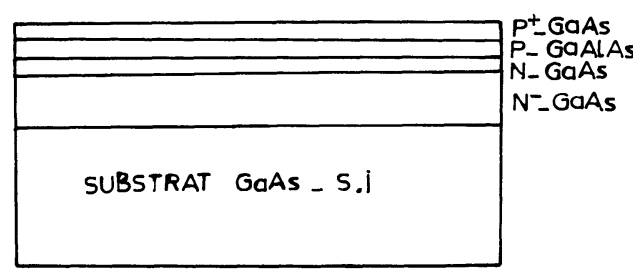

1 - LES DIFFERENTES COUCHES EPITAXIEES



2 - ATTAQUE SEIECTIVE DE LA COUCHE

$\mathrm{P}^{+}$GaAs SUR Ga $0.6^{\mathrm{Al}} 0.4^{\mathrm{As}}$

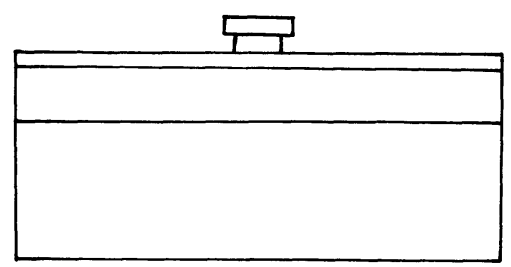

3 - ATTAQUE SELECTIVE DE LA COU-

-CHE P ${ }^{+} \mathrm{Ga}_{0.6^{\mathrm{Al}}}{ }_{0.4^{\mathrm{As}} \text { SUR GaAs }}$

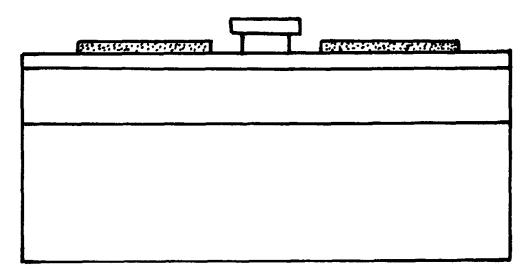

4 - METALLISATION AuGe+Ti

+ AuZn DE LA COUCHE N-GaAs

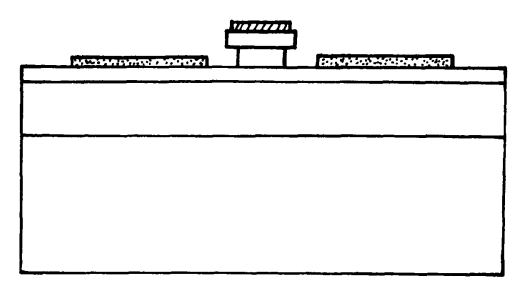

5 - METALLISATION AuZn SUR LA

COUCHE $\mathrm{P}^{+}$GaAs

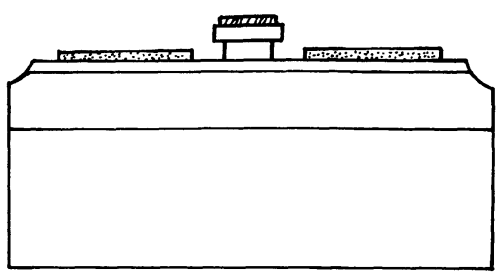

6 - ATTAQUE "MESA" POUR

DELIMITER LE COMPOSANT

Fig. 3. - Vue en coupe du composant après les principales étapes de fabrication : épitaxies, gravure et métallisations.

[Schematic cross-section of proposed device after epitaxy, etching and metallization steps.] 
3.2 PARTICULARITÉS DE LA PHOTOLITHOGRAPHIE ET OBSERVATION DU COMPOSANT. - La gravure du contact $\mathrm{p}^{+} \mathrm{GaAs}$ est effectuée à l'aide de la solution $\mathrm{NH}_{4} \mathrm{OH}+\mathrm{H}_{2} \mathrm{O}_{2}(30 \%)$ présentant un $\mathrm{pH}$ égal à 8,4 . Le temps d'attaque de l'ordre de $30 \mathrm{~s}$ se poursuit jusqu'à l'observation de la face supérieure du GaAlAs qui apparaît polie. Lorsque la gravure du GaAs est terminée, le matériau GaAlAs s'oxyde donnant une teinte sombre.

La dissolution du matériau p- $\mathrm{Ga}_{0.6} \mathrm{Al}_{0.4} \mathrm{As}$ sur les zones extérieures de la grille et de la photodiode est faite dans la solution $\mathrm{HF}$ concentré à une température égale à $50^{\circ} \mathrm{C}$. Le temps d'attaque de la couche de $0,5 \mu \mathrm{m}$ est égal à $45 \mathrm{~s}$ environ. La gravure du composé ternaire est terminée lorsque la surface de la couche n$\mathrm{GaAs}$ présente les lignes de ménisque caractéristiques du matériau binaire. Les conditions de température précédentes donnent un bon état de surface [8].

Les opérations de photogravure des métallisations s'effectuent à partir de la technique du « lift-off » qui maintient le bon état de la surface. Le processus technologique consiste à attaquer sélectivement d'abord la couche $\mathrm{p}^{+} \mathrm{GaAS}$ sur $\mathrm{p}-\mathrm{GaAlAs}$ ensuite la région $\mathrm{p}$ $\mathrm{GaAlAs}$ sur $\mathrm{n}-\mathrm{GaAs}$. La résine est déposée sur l'ensemble de l'échantillon (Fig. 3-1). Nous révélons la résine sur les électrodes de source et de drain. Une évaporation des pellicules métalliques $\mathrm{AuGe}-\mathrm{Ti}-\mathrm{AuZn}$ est effectuée sur la totalité de la face avant. Le traitement dans l'acétone bouillant dissout la résine à l'extérieur de la source et du drain. Cette dernière phase de la métallisation sur la couche $\mathrm{n}-\mathrm{GaAs}$ se termine par le traitement thermique à $425^{\circ} \mathrm{C}$ sous $\mathrm{N}_{2} / \mathrm{H}_{2}$ pendant $30 \mathrm{~s}$ (Fig. 3-4). La deuxième phase de l'élaboration des contacts met aussi en œuvre la technique du " lif-off » : ouverture de la fenêtre sur le contact de grille suivi d'un dépôt d'AuZn et dissolution dans l'acétone bouillant (Fig. 3-5). Le contact ohmique de l'électrode $\mathrm{p}^{+} \mathrm{GaAs}$ est amélioré par un traitement thermique en ambiance de gaz $\mathrm{N}_{2} / \mathrm{H}_{2}$, à $425^{\circ} \mathrm{C}$ pendant $15 \mathrm{~s}$. Les principales étapes de fabrication du dispositif sont montrées sur la figure 3 .

\section{Etude de la caractérisation électrique.}

L'étude expérimentale repose sur les relevés d'une part des caractéristiques courant-tension de l'hétérojonction Grille-Source et d'autre part des réseaux $I_{\mathrm{DS}}\left(V_{\mathrm{DS}}\right)$ à $V_{\mathrm{GS}}$ constant et $I_{\mathrm{DS}}\left(\mathrm{V}_{\mathrm{GS}}\right)$ à $V_{\mathrm{DS}}$ donné. L'analyse des caractéristiques de sortie et de transfert donne la valeur de la transconductance, de la tension de seuil et des résistances d'accès des électrodes de source et de drain.

4.1 Courant de SATURATION DE LA Diode GriLLE-SOURCE. - L'étude de la caractéristique couranttension de l'hétérojonction entre la grille et la source montre un courant de saturation inférieur à $1 \mu \mathrm{A}$ pour une tension inverse égale à $-3 \mathrm{~V}$. La résistance différentielle $\Delta V_{\mathrm{GS}} / \Delta I_{\mathrm{GS}}$ de la diode polarisée en inverse est identifiée, sa valeur est de l'ordre de $200 \mathrm{k} \Omega$.

4.2 CARACTÉRISTIQUES DU TRANSISTOR TEC. Nous considérons les caractéristiques du courant drain en fonction de la tension $V_{\text {DS }}$ pour des tensions de grille variant de 0 à $-3 \mathrm{~V}$. Le réseau $I_{\mathrm{DS}}\left(V_{\mathrm{DS}}\right) \mathrm{du}$ composant PI6 est donné sur la figure 4. Ces caractéris-

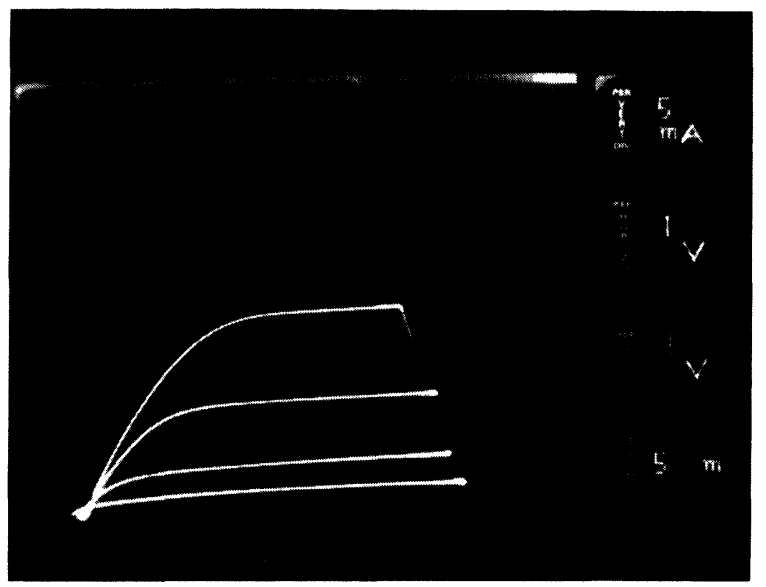

Fig. 4. - Réseau des caractéristiques $I_{\mathrm{DS}}\left(V_{\mathrm{DS}}\right)$ typique du composant JFET présentant un rapport longueur sur largeur de la grille égal à 0,02 . Echelles : $I_{\mathrm{DS}}, 5 \mathrm{~mA} / \mathrm{div}, V_{\mathrm{DS}}, 1 \mathrm{~V} / \mathrm{div}$ et $V_{\mathrm{GS}}, 1 \mathrm{~V}$ par pas.

[Typical $I_{\mathrm{DS}}\left(V_{\mathrm{DS}}\right)$ characteristics of an JFET with gate length/width of 0.02 . Scales : $I_{\mathrm{DS}}, 5 \mathrm{~mA} / \mathrm{div}, V_{\mathrm{DS}}, 1 \mathrm{~V} / \mathrm{div}$ and $V_{\mathrm{GS}}, 1 \mathrm{~V}$ per step.]

tiques sont typiques des mesures relevées sur nos dispositifs. Le courant de saturation $I_{\text {DSs }}$ est égal à $24 \mathrm{~mA}$ pour une tension de saturation de $4 \mathrm{~V}$. L'échantillon PI6 présente une tension de pincement de l'ordre de $5,95 \mathrm{~V}$. La figure 5 montre la caractéristique de transfert $I_{\mathrm{DS}}\left(V_{\mathrm{GS}}\right)$ mesurée pour une tension $V_{\mathrm{DS}}=5 \mathrm{~V}$.

Le relevé des variations $I_{\mathrm{DS}}\left(V_{\mathrm{GS}}\right)$ pour des tensions de drain inférieures ou égales à $100 \mathrm{mV}$ montre des variations linéaires [9]. Les droites coupent l'axe des $V_{\mathrm{GS}}$ en un point définissant la valeur de la tension de seuil. L'échantillon PI6 présente une tension égale à $V_{\mathrm{T}}=-4,6 \mathrm{~V}$.

4.3 VALEURS DES PARAMÉTRES ÉLECTRIQUES. L'étude des caractéristiques courant-tension donne les valeurs suivantes des paramètres électriques. Le courant de saturation de l'ensemble grille-photodiode est inférieur à $1 \mu \mathrm{A}$ pour une tension $V_{\mathrm{GS}}$ prise égale à $-3 \mathrm{~V}$. 


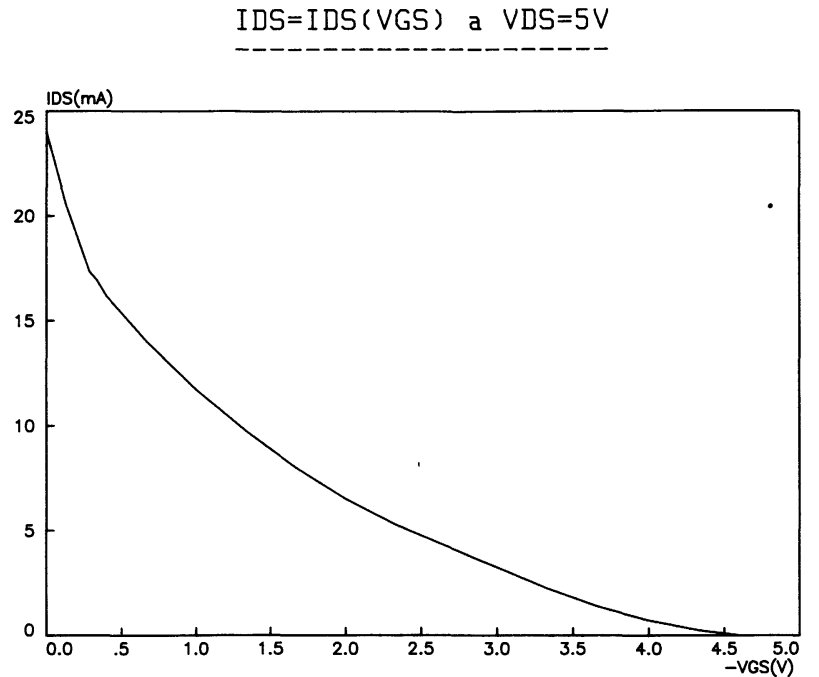

Fig. 5. - Caractéristique de transfert $I_{\mathrm{DS}}\left(V_{\mathrm{DS}}\right)$ mesurée pour une tension $V_{\text {DS }}=5 \mathrm{~V}$.

$\left[I_{\mathrm{DS}}\left(V_{\mathrm{DS}}\right)\right.$ transfert characteristic measured for a drain voltage of $5 \mathrm{~V}$.]

La transconductance déduite du réseau de la figure 4 est égale à $12 \mathrm{~mA} / \mathrm{V}$ pour les tensions $V_{\mathrm{GS}}=-1 \mathrm{~V}$, $V_{\mathrm{DS}}=5 \mathrm{~V}$ et une variation de tension de grille égale à $0,2 \mathrm{~V}$. Si la variation de $V_{\mathrm{GS}}$ est relevée à partir de la tension $V_{\mathrm{GS}}=0 \mathrm{~V} \cdot g_{\mathrm{m}}$ atteint la valeur de $15 \mathrm{~mA} / \mathrm{V}$.

La valeur de la transconductance par unité de largeur de la grille se situe à $60 \mathrm{mS} / \mathrm{mm}$.

La mesure des courants $I_{\mathrm{DS}}$ en fonction de la tension $V_{\mathrm{DS}}$ à faible niveau pour différentes valeurs de $V_{\mathrm{GS}}$ donne les valeurs de la résistance $r_{\text {Don }}$. Les valeurs expérimentales de $r_{\text {Don }}$ en fonction de la tension de grille sont reportées dans le tableau de la figure 6 . La variation de $r_{\text {Don }}$ est linéaire en fonction de la quantité $\left[1-\frac{\left(V_{\mathrm{bi}}-V_{\mathrm{GS}}\right)}{V_{\mathrm{P}}}\right]^{-1}$; la pente de la droite est égale à $\frac{L}{q N_{\mathrm{D}} Z a \mu_{\mathrm{n}}}[10]$. A partir des valeurs du tableau de la figure 6 , de la tension de diffusion $V_{\mathrm{bi}}=1,35 \mathrm{~V}$ et de l'épaisseur $a$ du canal :

$$
V_{\mathrm{P}}=\frac{q N_{\mathrm{D}} a^{2}}{2 \varepsilon_{0} \varepsilon_{\mathrm{r}}}
$$

$\left(N_{\mathrm{D}}<N_{\mathrm{A}}\right.$ et $\varepsilon_{\mathrm{r}}$ est la constante diélectrique relative du $\mathrm{GaAs}$ ), nous déduisons une mobilité de $3850 \mathrm{~cm}^{2} / \mathrm{V}$.s. Cette valeur de la mobilité est comparable à celle donnée dans la littérature. Nous estimons la somme des résistances d'accès de source $R_{\mathrm{s}}$ et de drain $R_{\mathrm{D}}$ égale à $40 \Omega$ [11]. Dans ces conditions, la transconductance intrinsèque pour $V_{\mathrm{GS}}=-1 \mathrm{~V}$ devient $15,8 \mathrm{~mA} / \mathrm{V}$ et a une valeur par unité de largeur de $80 \mathrm{mS} / \mathrm{mm}$.

\begin{tabular}{|c|c|c|c|c|}
\hline$V_{\mathrm{GS}}(\mathrm{V})$ & 0 & $-0,5$ & -1 & $-1,5$ \\
\hline$r_{\mathrm{Don}}(\Omega)$ & 100 & 111 & 125 & 150 \\
\hline
\end{tabular}

Fig. 6. - Tableau des valeurs mesurées de $r_{\mathrm{Don}}=\frac{\Delta V_{\mathrm{DS}}}{\Delta I_{\mathrm{DS}}}$ en fonction de $V_{\text {GS }}$ pour les faibles niveaux de $I_{\mathrm{DS}}$ dans la région ohmique $\left(V_{\mathrm{T}}=-4.6 \mathrm{~V}\right.$ à partir des résultats de la Fig. 5).

[Table showing the measured values of $r_{\mathrm{Don}}=\frac{\Delta V_{\mathrm{DS}}}{\Delta I_{\mathrm{DS}}}$ versus $V_{\mathrm{GS}}$ for low levels of $I_{\mathrm{DS}}$ in ohmic region $\left(V_{\mathrm{T}}=-4.6 \mathrm{~V}\right.$ from the results in Fig. 5).]

\section{Conclusion.}

Nous venons de décrire le principe de fonctionnement et la conception d'un dispositif JFET en vue de son association à une jonction PIN détectrice. Un des avantages du circuit est d'obtenir un courant de sortie proportionnel à la valeur de la transconductance gm [12]. Il est donc important de réaliser un TEC à $g_{\mathrm{m}}$ élevé.

Pour atteindre cet objectif, nous avons réalisé le composant actif à l'aide des croissances des couches n$\mathrm{GaAs}$ et $\mathrm{p}-\mathrm{Ga}_{0,6} \mathrm{Al}_{0,4} \mathrm{As}$. Nous avons détaillé les conditions d'élaboration en ne retenant que les particularités qui permettent la réussite et la reproductibilité de la réalisation de la structure.

Nous avons décrit les précautions à prendre et l'organisation des différentes opérations de gravure des électrodes de source et de drain d'une part et la définition de la grille, d'autre part. L'étude expérimentale a montré les caractéristiques courant-tension de sortie et les courbes de transfert. $I_{\mathrm{DS}}\left(\mathrm{V}_{\mathrm{GS}}\right)_{V_{\mathrm{DS}}}$. Ensuite, l'analyse des réseaux $I_{\mathrm{DS}}\left(V_{\mathrm{DS}}\right)_{V_{\mathrm{GS}}}$ précédents a permis de déduire des valeurs de la transconductance supérieures ou égales à $12 \mathrm{~mA} / \mathrm{V}(60 \mathrm{mS} / \mathrm{mm})$. Enfin, l'étude des variations du courant drain-source pour des valeurs petites de $I_{\mathrm{DS}}$ et des polarisations basses de $V_{\mathrm{DS}}$ montrent respectivement une tension de seuil à $-4,6 \mathrm{~V}$ et des résistances $r_{\text {Don }}$ comprises entre 100 et $150 \Omega$ lorsque $V_{\mathrm{GS}}$ varie. Les résistances d'accès des électrodes de source et de drain sont égales à $40 \Omega$. Parallèlement la mobilité des électrons dans le canal est comparable aux valeurs mesurées sur les transistors à effet de champ fabriqués dans d'autres laboratoires.

\section{Remerciements.}

Les auteurs remercient Monsieur D. Esteve pour ses conseils et Messieurs R. Leguerre et A. Martinez pour les discussions à propos du dispositif. 


\section{Bibliographie}

[1] Wada, O., Miura, S., Ito, M., FujiI, T., Sakurai, T. et Hiyamizu, S., Appl. Phys. Lett. 42 (4) (1983) 380.

[2] Kolbas, R. M., Abrokwah, J., Carney, J. K., Bradshaw, D. H., Elmer, B. R. et BIARD, J. R., Appl. Phys. Lett. 43 (9) (1983) 821.

[3] Ito, M., Wada, O., Nakai et SaKurai, T., IEEE Electron Device Lett., EDL-5 (1984) 531.

[4] Carter, A. C., Forles, N. et Goodfellow, R. C., Electron. Lett. 18 (2) (1982) 72.

[5] Abrokwah, J. K., Hitchell, M. L., Borrel, J. E. et SChulze, D. R., J. Electron. Mat. 10 (4) (1981) 723.

[6] Nanishi, Y., Japan J. Appl. Phys. 17 (7) (1978) 1177.

[7] Therez, F., Chikouche, A., Alcubilla, R. et Bielle-Daspet, D., ESA SP-210 (11) (1984) 95.

[8] Morkoc, H., Bandy, S. G., SANKaran, R., ANTYPAS, G. A. et BELL, R., IEEE Trans. Electron Devices 25 (6) (1978) 619.
[9] WhOLEY, J., OMORI, Gallium arsenide and related compounds (St Louis) (1978), Inst. Phys. Conf. 45 (1979) 270.

[10] Soares, R., Craffeuil, J. et Obrégon, J., Applications of GaAs MESFETS (Artech House Inc.) 1983 ;

Combes, P. F., Graffeuil, et Sautereau, J. F., Composants, dispositifs et circuits actifs pour micro-ondes (Dunod, Bordas, Paris, ISBN 2-04015750-G) 1985.

[11] Baier, S. M., Shur, M. S., Lee, K., Cirillo, N. C. et HANKA, S. A., IEEE Trans. Electron Devices 32 (12) (1985) 2824.

[12] Makiuchi, M., Hamaguchi, H., Kumai, T., Ito, M., WADA, O. et SAKURAI, T., IEEE Electron Device Lett. 6 (12) (1985) 634. 